\title{
Dynamical Electroweak Symmetry Breaking in String Models with D-branes
}

\author{
Noriaki Kitazawa \\ Department of Physics, Tokyo Metropolitan University, \\ Hachioji, Tokyo 192-0397, Japan \\ e-mail:kitazawa@phys.metro-u.ac.jp
}

\begin{abstract}
The possibility of dynamical electroweak symmetry breaking by strong coupling gauge interaction in models with D-branes in String Theory is examined. Instead of elementary scalar Higgs doublet fields, the gauge symmetry with strong coupling (technicolor) is introduced. As the first step of this direction, a toy model, which is not fully realistic, is concretely analyzed in some detail. The model consists of D-branes and anti-D-branes at orbifold singularities in $\left(T^{2} \times T^{2} \times T^{2}\right) / \mathbf{Z}_{3}$ which preserves supersymmetry. Supersymmetry is broken through the brane supersymmetry breaking. It is pointed out that the problem of large $S$ parameter in dynamical electroweak symmetry breaking scenario may be solved by natural existence of kinetic term mixings between hypercharge $\mathrm{U}(1)$ gauge boson and massive anomalous $\mathrm{U}(1)$ gauge bosons. The problems to be solved toward constructing more realistic models are clarified in the analysis.
\end{abstract}




\section{Introduction}

The quest for natural mechanism of electroweak symmetry breaking is one of the most important subject in high energy physics. Supersymmetric extension of the standard model [1, 2, 3, 4, 5, 6] is the most popular scenario because of smooth transition into the grand unification with radiative electroweak symmetry breaking [7]. Instead of supersymmetry, some global symmetries also can act the role to stabilize the electroweak symmetry breaking scale [8, 9]. The other popular scenario is assuming extra space dimensions [10, 11, 12] by which Planck scale of gravity is reduced to the scale of electroweak symmetry breaking. The existence of extra dimensions also makes possible to break gauge symmetry by boundary conditions, which is utilized to "unify" Higgs doublet fields and gauge fields [13, 14, 15. or to break electroweak symmetry without Higgs doublet fields [16. The possibility of dynamical electroweak symmetry breaking [17, 18, 19, 20, 21, 22, 23] is still pursued in spite of that precision electroweak data put this possibility at a disadvantage (see Ref.[24] for a review).

In phenomenological efforts in String Theory, many works have focused on providing appropriate boundary conditions at high energies for successful supersymmetric extensions of the standard model or supersymmetric grand unified theories in field theory without theoretical inconsistencies. Flux compactifications for moduli stabilizations can give warped extra dimensions and natural electroweak symmetry breaking [25], but technical difficulties do not allow explicit quantitative analysis in String Theory. Although some scenarios utilizing "stringy" effects, Higgs doublet fields as tachyons in D-brane recombinations [26, 27], symmetry breaking by string loop corrections [28, 29], for example, have been proposed, technical difficulties prevent to construct concrete calculable models.

In this paper we focus on models with D-branes in type II string theory. There are two types of models: models with D-branes intersecting in compact space [30, 31, 32, 26, 33, 34, 35, 36, 37, 38] and models with D-branes at singularities in compact space [39]. In both types of models there is a tendency for the number of Higgs doublet fields to be many. It was shown that the number of (massless) Higgs doublet fields in the first type of models should be larger than three [40], if we consider simple factorized toroidal compactifications $T^{2} \times T^{2} \times T^{2}$ with some orbifold or orientifold projections. (This is not the case, if Higgs doublet fields come from stretched open string and originally massive [34]. More general compactifications also change the situation [41]. Introduction of the compositeness may also change the situation [42, 43, 44].) The similar situation happens in the second type of models.

In case with extra Higgs doublet fields, it is very difficult to escape from too large FCNC to be consistent with experiments. If all the Higgs doublet fields get vacuum expectation values, the Yukawa coupling matrices of massive neutral scalar fields are not diagonal in mass eigenstates of quarks and leptons, and these neutral scalar fields mediate large FCNC. It is very unnatural to assume very large masses for these neutral scalar fields. Even if only one Higgs doublet fields gets vacuum expectation value, the situation is the same without assuming very large masses for extra Higgs doublet fields. This fact does not depend on whether low-energy supersymmetry exists or not.

In case of low string scale with large compact dimensions for radiative electroweak symmetry breaking by string loop corrections, massive string modes associated with extra 
Higgs doublet fields also mediate large FCNC. It can be shown that the four-fermion processes mediated by Higgs exchanges are also dressed by string form factors in the similar way that is shown in Ref. [45] on the processes mediated by gauge boson exchanges. The lower bound on the string scale $2 \sim 3 \mathrm{TeV}$ is obtained in Ref.[45], which is critical for radiative electroweak symmetry breaking. In the models of the first type there are further additional sources of FCNC by the world-sheet instanton effect which is necessary to generate Yukawa couplings for the masses of quarks and leptons. A strong constraint on the string scale to be larger than about $10^{3} \mathrm{TeV}$ is obtained in Ref.[46] assuming simple compactifications which make models calculable. Therefore, radiative breaking is not the possibility for the first type of models. Astrophysical constraints on the sizes of extra dimensions (we will discuss this constraint in section 3.1 in detail) almost excluded the possibility of the string scale of a few $\mathrm{TeV}$ and of radiative breaking scenario even in the second type of models 1 .

It looks that the safest way to proceed is to find the models with minimal number of Higgs doublet fields with low-energy supersymmetry (and its breaking), and to provide appropriate boundary conditions for standard radiative electroweak symmetry breaking taking the string scale very high. In fact there are many efforts in this direction with moduli stabilization (for a resent effort, see Ref.[48, for example). There is, however, a possibility of dynamical electroweak symmetry breaking by some strong gauge interaction which has not seriously examined yet. It is well known that this scenario, technicolor, is strongly constrained by the precise experimental measurements of the corrections to selfenergies of electroweak gauge bosons. Though this problem of large $S$ parameter is fatal for simple technicolor dynamics like QCD scaling-up, it has been proposed that certain non-trivial dynamics do not cause the problem [49, 50, 51]. It is also pointed out that the existence of massive vector fields which mix with hypercharge gauge boson in kinetic term (kinetic term mixing) may solve the problem, even if the dynamics is simple [52, 53]. We focus on the second possibility, since massive anomalous $U(1)$ gauge fields which can have kinetic term mixings with hypercharge gauge field naturally emerge in models with D-branes. The aim of this paper is not to propose a realistic model, but to examine the possibility by analyzing a simple concrete toy model, though it is non-realistic. The model is based on D-branes at orbifold singularities with a supersymmetric $\left(T^{2} \times T^{2} \times T^{2}\right) / \mathbf{Z}_{3}$ compactification. Supersymmetry is broken at the string scale by introducing anti-Dbranes, namely by the brane supersymmetry breaking [54, 55, 56, [57, 58], and it is assumed that all the originally massless scalar modes obtain masses of the order of the string scale through the tree-level closed string exchanges [47]. We also assume that gauginos obtain masses of the order of the string scale, though some bulk supersymmetry breaking would

\footnotetext{
1 There are two types of massless scalar modes on a D-brane at a singularity: one is associated with D-brane moduli and the other is associated with Wilson line [47. The scale of mass correction for the first type is typically one order lower than the string scale due to the contributions from twisted closed strings. Though the scale of mass correction for the second type is originally governed by the compactification scale (and can be smaller than the string scale), we have to use orbifold projection to extract scalar fields in non-adjoint representations (Higgs doublet fields). Then the scale of mass correction is again one order lower than the string scale, because the contributions from twisted closed strings dominate. The mass corrections to the massless scalar modes from the open strings between different D-branes have not investigated yet.
} 
have to be introduced [59, 60. 2

This paper is organized as follows. In section 2 the scenario of dynamical electroweak symmetry breaking in field theory is briefly reviewed. The problem of large $S$ parameter is also reviewed. In section 3 a toy model is constructed and investigated. The anomaly structure and masses of U(1) gauge fields are investigated in detail. The contributions to $S$ parameter through the anomalous U(1) gauge boson exchanges are quantitatively estimated, and the possibility of dynamical electroweak symmetry breaking in String Theory is examined. In section 4 some general comments and conclusions are presented.

\section{Dynamical electroweak symmetry breaking}

We very briefly review technicolor theory [62] and its problems with precision electroweak data.

Consider the standard model without Higgs doublet field. The chiral symmetry in quark sector would be spontaneously broken by the quark pair condensates due to the strong coupling effect of QCD. Since electroweak gauge symmetry would be also broken at the same time, the mass of the weak bosons would be of the order of $10 \mathrm{MeV}$ corresponding to the QCD scale $\Lambda_{\mathrm{QCD}} \sim 100 \mathrm{MeV}$ where QCD coupling becomes order one: $\alpha_{s} \sim 1$. Though electroweak gauge symmetry would be broken, leptons would not obtain masses because of no mediation of the breaking to lepton sector.

Introduce additional QCD-like gauge symmetry, $\mathrm{SU}\left(N_{\mathrm{TC}}\right)$, with additional left-handed fermions ("techni-fermions")

$$
\begin{aligned}
\left(\begin{array}{c}
N_{L} \\
E_{L}
\end{array}\right) & \sim\left(N_{\mathrm{TC}}, 2\right)_{0}, \\
\left(N_{R}\right)^{c} & \sim\left(N_{\mathrm{TC}}^{*}, 1\right)_{-1 / 2}, \\
\left(E_{R}\right)^{c} & \sim\left(N_{\mathrm{TC}}^{*}, 1\right)_{+1 / 2}
\end{aligned}
$$

under $\mathrm{SU}\left(N_{\mathrm{TC}}\right) \times \mathrm{SU}(2)_{L} \times \mathrm{U}(1)_{Y}$. All the gauge anomaly is canceled out $\left(N_{\mathrm{TC}}\right.$ should be even for $\mathrm{SU}(2)_{L}$ global anomaly cancellation). Suppose $\mathrm{SU}\left(N_{\mathrm{TC}}\right)$, namely technicolor, becomes strong at the scale $\Lambda_{\mathrm{TC}} \sim 1 \mathrm{TeV}$, we expect the condensates

$$
\left\langle\bar{N}_{R} N_{L}\right\rangle=\left\langle\bar{E}_{R} E_{L}\right\rangle \neq 0
$$

by the criteria of maximal remaining gauge symmetry. This causes electroweak symmetry breaking and weak bosons obtain masses of the order of $100 \mathrm{GeV}$. The masses of ordinary fermions can be produced through four-fermion interactions like

$$
\mathcal{L}_{\mathrm{f}-\mathrm{mass}}=-\frac{1}{M_{u E}^{2}}\left(\bar{q}_{L}^{i} u_{R}\right)\left(\bar{N}_{R} L_{L i}\right)-\frac{1}{M_{u N}^{2}}\left(\bar{q}_{L}^{i} u_{R}\right) \epsilon_{i j}\left(\bar{L}_{L}^{j} E_{R}\right)+\text { h.c. }
$$

for the up quark, where $q_{L}=\left(u_{L} d_{L}\right)^{T}, L_{L}=\left(N_{L} E_{L}\right)^{T}$ and $M_{u E}$ and $M_{u N}$ are scales of the physics which mediate electroweak symmetry breaking to the up quark. The flavor physics

\footnotetext{
${ }^{2}$ For model building with different philosophy using AdS/CFT correspondence, see Ref. 61, for example.
} 
has direct connection to the origin of these kinds of four-fermion interactions among ordinary fermions and techni-fermions. This is the scenario of (one-doublet) technicolor for natural electroweak symmetry breaking with dimensional transmutation.

Precision electroweak measurements give constraints on vacuum polarizations of weak bosons, $W^{ \pm}$and $Z^{0}$ and photon, or $W_{1,2,3}$ and $B$ corresponding to $\mathrm{SU}(2)_{L}$ and $\mathrm{U}(1)_{Y}$. At low energies, where we can neglect all the higher derivative terms in the effective action, it can be shown that only three parameters are required to describe the new physics contributions beyond the standard model by virtue of symmetry and renormalization [63, 64, 65, 66]. Those three parameters are defined as

$$
\begin{aligned}
S & =-16 \pi \Pi_{3 Y}^{\prime}(0), \\
T & =\frac{4 \pi}{s^{2} c^{2} m_{Z}^{2}}\left[\Pi_{11}(0)-\Pi_{33}(0)\right], \\
U & =16 \pi\left[\Pi_{11}^{\prime}(0)-\Pi_{33}^{\prime}(0)\right],
\end{aligned}
$$

where $s=\sin \theta_{W}$ and $c=\cos \theta_{W}$ with Weinberg angle $\theta_{W}$, and the vacuum polarizations with no Lorentz indices are defined as

$$
\Pi_{A B}^{\mu \nu}(q)=\left(g^{\mu \nu}-\frac{q^{\mu} q^{\nu}}{q^{2}}\right) \Pi_{A B}\left(q^{2}\right), \quad \Pi_{A B}^{\prime}\left(q^{2}\right) \equiv \frac{d \Pi_{A B}\left(q^{2}\right)}{d q^{2}}
$$

with $A, B=1,2,3, Y$ corresponding to $W_{1,2,3}$ and $B$. These vacuum polarizations are defined so that they include unknown new physics effects only (the known contributions from the standard model are subtracted). The vacuum polarizations at zero momentum describe the corrections to the masses, and the derivative of the vacuum polarizations at zero momentum describe the corrections to kinetic terms. We can understand that $S$ parameter describes kinetic term mixing between $W_{3}$ and $B$ through new physics beyond the standard model.

Since technicolor and techni-fermions are new physics, they are constrained through the above three parameters. The contribution to $S$ parameter can be naively estimated by the calculation of techni-fermion one-loop diagrams neglecting strong coupling technicolor effects:

$$
S=\frac{N_{\mathrm{TC}}}{6 \pi} N_{D}
$$

where $N_{D}$ is the number of $\mathrm{SU}(2)_{L}$ doublets of techni-fermions $\left(N_{D}=1\right.$ in the present case). There are estimates for QCD-like technicolor including the effects of strong coupling dynamics using the technique of scaling up experimental data of real QCD phenomena, and the results support that the above simple estimate is rather good. The experimental constraint, assuming Higgs mass $600 \mathrm{GeV}$ and $S>0$ (which is appropriate for QCD-like technicolor dynamics), is given as $S<0.09$ at 95\% CL [67], and one can conclude that the technicolor scenario with QCD-like technicolor dynamics has already been excluded. 3

It should be mentioned that experimental constraints on $T$ parameter also make the situation of technicolor scenario unfavorable, if the physics of ordinary fermion mass

\footnotetext{
${ }^{3}$ Some simple models passing this constraint have been proposed in Refs. 68, 69, 70].
} 
generation is considered. Since top quark is much heavier than bottom quark, "nonoblique corrections", which can not be parametrized by the above three parameters, tend to become large as well as $T$ parameter. This is another difficulty of technicolor scenario, which is strongly related with the physics of the mediation of electroweak symmetry breaking to quarks and leptons (namely, the origin of flavor physics). In this paper we concentrate on $S$ parameter problem leaving flavor problems for future researches.

\section{Technicolor in D-brane models}

In the following subsections, we construct a toy model using D-branes at orbifold singularities, investigate the anomaly structure and masses of anomalous U(1)'s, and estimate their contributions to $S$ parameter.

\subsection{A toy model}

We consider a simple compact space of $\left(T^{2} \times T^{2} \times T^{2}\right) / \mathbf{Z}_{3}$ with orbifold projection vector $v=(1 / 3,1 / 3,-2 / 3)$. There are three orbifold singularities in each torus, and they can be specified as $\mathrm{I}_{i}, \mathrm{II}_{i}$ and $\mathrm{III}_{i}$ for torus $i=1,2,3$. There are 27 singular points in total in this compact space, and we can specify one of them as $\left(\mathrm{I}_{1}, \mathrm{II}_{2}, \mathrm{III}_{3}\right)$, for example. The model includes D3-branes, D7 ${ }_{i}$-branes and their anti-branes, where a $\mathrm{D} 7_{i}$-brane means a D7-brane filling compact space except for $i$-th torus.

First, we construct a local model at $\left(\mathrm{I}_{1}, \mathrm{I}_{2}, \mathrm{I}_{3}\right)$ with brane supersymmetry breaking. We would like to have an asymptotically free gauge symmetry (assuming all scalar fields are decoupled) in addition to the gauge symmetry of the standard model. Introduce D3, $\mathrm{D} 7_{1}, \mathrm{D} 7_{2}, \mathrm{D} 7_{3}$ and $\overline{\mathrm{D}}_{1}$ branes with the following transformation matrices on Chan-Paton factors under $\mathbf{Z}_{3}$ transformation:

$$
\begin{aligned}
\gamma_{3} & =\operatorname{diag}\left(\mathbf{1}_{3}, \alpha \mathbf{1}_{2}, \alpha^{2} \mathbf{1}_{1}\right) \\
\gamma_{7_{1}} & =\gamma_{7_{2}}=\gamma_{7_{3}}=\operatorname{diag}\left(0,0, \alpha^{2} \mathbf{1}_{1}\right) \\
\gamma_{\overline{7}_{1}} & =\operatorname{diag}\left(\mathbf{1}_{3}, 0,0\right)
\end{aligned}
$$

where $\mathbf{1}_{a}$ means an $a \times a$ unit matrix and $\alpha=\exp (i 2 \pi / 3)$. The twisted Ramond-Ramond $(\mathrm{R}-\mathrm{R})$ tadpoles are canceled by satisfying the condition

$$
3 \operatorname{Tr} \gamma_{3}+\sum_{i=1,2,3} \operatorname{Tr} \gamma_{7_{i}}-\sum_{i=1,2,3} \operatorname{Tr} \gamma_{\overline{7}_{i}}=0
$$

The total gauge symmetry is $\mathrm{U}(3) \times \mathrm{U}(2) \times \mathrm{U}(1)$ on D3-brane, $\mathrm{U}(1)_{i}^{\prime}$ on $\mathrm{D} 7_{i}$-branes $(i=$ $1,2,3)$, and $\mathrm{U}(3)$ on $\overline{\mathrm{D}}_{1}$-brane. The color $\mathrm{SU}(3)_{c}$ and weak $\mathrm{SU}(2)_{L}$ are identified as $\mathrm{SU}(3)$ in $\mathrm{U}(3)=\mathrm{SU}(3) \times \mathrm{U}(1)_{3}$ and $\mathrm{SU}(2)$ in $\mathrm{U}(2)=\mathrm{SU}(2) \times \mathrm{U}(1)_{2}$ on D3-branes, respectively. The hypercharge $\mathrm{U}(1)_{Y}$ is defined as

$$
\frac{Y}{2} \equiv-\left(\frac{1}{3} Q_{3}+\frac{1}{2} Q_{2}+Q\right)
$$

where $Q_{3}, Q_{2}$ and $Q$ denote the charges of $\mathrm{U}(1)$ symmetries on D3-brane. This is a simple realization of non-anomalous hypercharge gauge symmetry in many possibilities [41]. In 
the following we will see that this simple realization can not give realistic lepton spectrum, because three "higgsinos" remain after decoupling of all the scalars. Since the aim of this paper is to examine the possibility of technicolor with anomalous U(1)'s using a simple toy model, we accept this unrealistic situation. The technicolor gauge symmetry $\mathrm{SU}(3)_{T C}$ is identified as $\mathrm{SU}(3)$ in $\mathrm{U}(3)=\mathrm{SU}(3) \times \mathrm{U}(1)_{T C}$ on $\overline{\mathrm{D}}_{1}$-brane.

The value of gauge coupling at the string scale depends on the compactification scale in general. From Dirac-Born-Infeld action (with $\kappa_{10}^{2}=(2 \pi)^{7}\left(\alpha^{\prime}\right)^{4} / 2$ in type IIB theory) we obtain gauge couplings for each D-brane as

$$
\begin{aligned}
g_{D 3} & =\sqrt{2 \pi g_{s}} \\
g_{D 7_{1}}=g_{\overline{D 7_{1}}} & =\sqrt{2 \pi g_{s}}\left(\frac{2 \pi \sqrt{\alpha^{\prime}}}{2 \pi r}\right)^{2}, \\
g_{D 7_{2}}=g_{D 7_{3}} & =\sqrt{2 \pi g_{s}}\left(\frac{2 \pi \sqrt{\alpha^{\prime}}}{2 \pi r}\right)\left(\frac{2 \pi \sqrt{\alpha^{\prime}}}{2 \pi R}\right),
\end{aligned}
$$

where $g_{s}=e^{\langle\phi\rangle}$ is the string coupling and $R$ and $r$ are the compactification radii for the first torus and second and third tori, respectively (the radii of second and third tori are taken to be the same, for simplicity). We assume $g_{D 3}=\sqrt{2 \pi g_{s}} \simeq 1$ and do not discuss the possibility to reproduce smaller gauge couplings of $\mathrm{SU}(2)_{L}$ and $\mathrm{U}(1)_{Y}$ than that of $\mathrm{SU}(3)_{c}$. Furthermore, we assume $r \simeq \sqrt{\alpha^{\prime}}$ for strong coupling technicolor at low energies, and assume large $R$ for low string scale of the order of $10 \mathrm{TeV}$ to have light anomalous $\mathrm{U}(1)$ gauge bosons. Since the relation between reduced Planck scale $M_{P}^{*}$ and the string scale $M_{s}^{*} \equiv 1 / 2 \pi \sqrt{\alpha^{\prime}}$ is given by

$$
M_{s}^{*}=\sqrt{\frac{g_{s}}{4 \pi}}\left(\frac{2 \pi \sqrt{\alpha^{\prime}}}{2 \pi R}\right)\left(\frac{2 \pi \sqrt{\alpha^{\prime}}}{2 \pi r}\right)^{2} M_{P}^{*},
$$

we have $R \simeq 0.02 \mu \mathrm{m} \simeq 5 \mathrm{eV}^{-1}$ which is not yet excluded by the supernova SN1987a constraint $R<0.066 \mu \mathrm{m}$ [71] 4. Six-dimensional gravitational scale is given by

$$
M_{P}^{6 \mathrm{D}}=\sqrt{M_{P}^{*} M_{s}^{*}}\left(\frac{2 \pi \sqrt{\alpha^{\prime}}}{2 \pi R}\right)^{1 / 2} \simeq 30 \mathrm{TeV}
$$

which is not yet excluded by the constraint $M_{P}^{6 \mathrm{D}}>14 \mathrm{TeV}$ 67]. The gauge couplings on $\mathrm{D} 7_{2}$ and $\mathrm{D} 7_{3}$ branes are very small of the order of $10^{-7}$.

Though twisted tadpole cancellation, eq.(14), guarantee the anomaly cancellation for gauge symmetry on D3-brane, gauge symmetries on D7-branes are anomalous without canceling twisted $\mathrm{R}-\mathrm{R}$ tadpoles at all the singular points in their world volumes. After all, twisted R-R tadpoles at all the 27 singularities should be canceled. Table 1 shows D-branes and anti-D-branes which concern twisted R-R tadpole cancellation at each 27 singularity. We do not consider untwisted R-R tadpole cancellation (cancellation of mixed

\footnotetext{
${ }^{4}$ Here, we do not consider stronger constraints from astrophysics assuming substantial branching ratios of decays of Kaluza-Klein gravitons to photon [72, 73, which is model dependent.
} 
gravitational anomalies) and moduli stabilization, since they would not be very essential for the results in this paper, though moduli stabilizations might be necessary to prevent pair annihilations between branes and anti-branes. Many D-branes and anti-D-branes are introduced with the following transformation matrices under $\mathbf{Z}_{3}$ transformation.

$$
\begin{aligned}
\gamma_{3^{\prime}} & =\gamma_{3^{\prime \prime}}=\operatorname{diag}\left(\mathbf{1}_{3}, \alpha \mathbf{1}_{2}, \alpha^{2} \mathbf{1}_{1}\right) \\
\gamma_{3^{(a)}} & =\operatorname{diag}\left(\mathbf{1}_{1}, 0,0\right), \quad a=1 \cdots 6 \\
\gamma_{\overline{3}^{(a)}} & =\operatorname{diag}\left(0,0, \alpha^{2} \mathbf{1}_{1}\right), \quad a=1 \cdots 6 \\
\gamma_{7_{1}^{\prime}} & =\gamma_{7_{1}^{\prime \prime}}=\operatorname{diag}\left(0,0, \alpha^{2} \mathbf{1}_{1}\right) \\
\gamma_{\overline{7}_{2}^{\prime}} & =\gamma_{\overline{7}_{2}^{\prime \prime}}=\operatorname{diag}\left(0,0, \alpha^{2} \mathbf{1}_{2}\right) \\
\gamma_{7_{3}^{\prime}} & =\gamma_{7_{3}^{\prime \prime}}=\operatorname{diag}\left(0,0, \alpha^{2} \mathbf{1}_{1}\right) .
\end{aligned}
$$

Most additional D-branes introduce additional U(1) gauge symmetries, except for D3', D3 ${ }^{\prime \prime}, \overline{\mathrm{D}}_{2}^{\prime}$ and $\overline{\mathrm{D}}_{2}^{\prime \prime}$ branes. Two copies of the standard model massless field contents are realized with each D3' and D3" brane, which have communication with our world on D3-brane at $\left(\mathrm{I}_{1}, \mathrm{I}_{2}, \mathrm{I}_{3}\right)$ singularity only through massive (heavier than the string scale 10 $\mathrm{TeV}$ ) open string modes and closed string modes (including gravity).

All the massless fermion modes at $\left(\mathrm{I}_{1}, \mathrm{I}_{2}, \mathrm{I}_{3}\right)$ singularity are listed in Table 2, All the non-Abelian gauge anomalies are canceled. We assume that all the massless scalar modes obtain masses of the order of the string scale and decouple from low energies through closed string tree-level effects (or open-string one-loop effects) in this configuration of brane supersymmetry breaking [47]. It is naively assumed that all the gauginos also obtain masses of the order of the string scale and decouple. Since gaugino Majorana mass is protected by approximate (discrete) R symmetry, to obtain large gaugino masses, introduction of bulk supersymmetry breaking à la Sherk-Schwarz would be required to appropriately break (discrete) $R$ symmetry [59, 60]. Technicolor gauge interaction is asymptotically free with the coefficient of beta function as $b_{\mathrm{TC}}=-13 / 3\left(b_{\mathrm{TC}}=-7 / 3\right.$ with techni-gluino $)$. The number of $\mathrm{SU}(2)_{L}$ doublets in techni-fermions is one, but the hypercharge assignment is different from that in one-doublet model which has been reviewed in the previous section. This is due to the fact that we have to have three "higgsinos" in this simple hypercharge realization on a D3-brane, and techni-fermions are necessary to cancel the anomaly of $\mathrm{U}(1)_{Y}$. The anomaly structure of $31 \mathrm{U}(1)$ gauge symmetry, including $\mathrm{U}(1)_{Y}$, is discussed in the next subsection.

While technicolor running coupling becomes large below the string scale, the coefficient of beta function is smaller in absolute value than that of QCD. If these gauge couplings are equal at the string scale, the scale of technicolor condensation is lower than QCD scale. Here, we simply assume large threshold corrections (Ref. [74, 75, 76, 77, 78] for type I theory, Ref.[79, 80, 81] for intersecting D-brane models, and Ref. [82, 83] for models with D-branes at singularities) by which technicolor coupling is about two times larger than QCD coupling at the string scale of the order of $10 \mathrm{TeV}$ (100\% correction). Then technicolor coupling becomes strong at the energy scale of the order of $100 \mathrm{GeV}$. This may not be so unreasonable. Because technicolor lives on D7-brane and QCD lives on D3-brane, only technicolor gauge coupling receives corrections by Kaluza-Klein states. It also has been pointed out in Ref. [82] that the effect of winding modes can be significant 
in case of large compactifications. Another possibility is taking compactification scale $r^{2}$ about two times smaller than the string scale. However, more natural realizations of strong coupling technicolor should be pursued in realistic models. One possibility is to realize technicolor on D3-branes and standard model gauge symmetry on D7-branes, though the realization of hypercharge becomes rather non-trivial.

The problem to look for the pattern of tech-fermion condensates, namely the problem of vacuum alignment, is a difficult issue in this kind of system with complicated interactions. Usually, it is assumed that the pattern with maximal symmetry is preferred. Here, we further assume that the only two fermions which can be at the same place in compact space can condensate. One preferred pattern is

$$
\begin{aligned}
& \left\langle\Psi_{L}^{I_{3}=-1 / 2} \Psi_{E}\right\rangle=\left\langle\Psi_{L}^{I_{3}=+1 / 2} \Psi_{7_{2}}\right\rangle \neq 0, \\
& \left\langle\Psi_{L}^{\prime I_{3}=-1 / 2} \Psi_{E}^{\prime}\right\rangle=\left\langle\Psi_{L}^{\prime I_{3}=+1 / 2} \Psi_{7_{3}^{\prime}}\right\rangle \neq 0, \\
& \left\langle\Psi_{L}^{\prime \prime I_{3}=-1 / 2} \Psi_{E}^{\prime \prime}\right\rangle=\left\langle\Psi_{L}^{\prime \prime} I_{3}=+1 / 2 \Psi_{7_{3}^{\prime \prime}}\right\rangle \neq 0, \\
& \left\langle\Psi_{7_{2}^{\prime}}^{I_{3}=-1 / 2} \Psi_{7_{1}}^{i=1}\right\rangle=\left\langle\Psi_{7_{2}^{\prime}}^{I_{3}=+1 / 2} \Psi_{7_{1}}^{i=2}\right\rangle \neq 0, \\
& \left\langle\Psi_{\overline{7}_{2}^{\prime \prime}}^{I_{3}=-1 / 2} \Psi_{7_{1}}^{i=3}\right\rangle=\left\langle\Psi_{\overline{7}_{2}^{\prime \prime}}^{I_{3}=+1 / 2} \Psi_{7_{3}}\right\rangle \neq 0 .
\end{aligned}
$$

The first two condensates trigger the electroweak symmetry breaking in very similar way in one-doublet model in previous section. The condensates of second and third lines result "electroweak symmetry breaking" in two mirror worlds.

\subsection{Anomalous U(1) gauge bosons}

There are $31 \mathrm{U}(1)$ gauge symmetries including $\mathrm{U}(1)_{Y}$. We examine the anomaly structure of seven $\mathrm{U}(1)$ gauge symmetries on five D-branes at a singularity $\left(\mathrm{I}_{1}, \mathrm{I}_{2}, \mathrm{I}_{3}\right.$ ). (The $\mathbf{Z}_{3}$ transformation matrices for Chan-Paton factors of those D-branes are given in eqs.(11), (12) and (13) ). Then, we investigate the mass matrix of whole U(1) gauge bosons.

First, consider mixed non-Abelian anomalies of seven $\mathrm{U}(1)$ gauge symmetries in Dbrane basis:

$$
q_{1}=Q_{T C}, \quad q_{2} \equiv Q_{3}, \quad q_{3} \equiv Q_{2}, \quad q_{4} \equiv Q, \quad q_{5}=Q_{1}^{\prime}, \quad q_{6}=Q_{2}^{\prime}, \quad q_{7}=Q_{3}^{\prime},
$$

where $Q_{T C}$ is the $\mathrm{U}(1)$ charge in $\mathrm{U}(3)_{T C}, Q_{i}^{\prime}$ are charges of $\mathrm{U}(1)_{i}^{\prime}$ from $\mathrm{D} 7_{i}$-branes with $i=1,2,3$. The generators of non-Abelian gauge symmetries are denoted as $\left(T^{A}\right)_{a}$ with $a=1,2,3$ for $\mathrm{SU}(3)_{T C}, \mathrm{SU}(3)_{c}$ and $\mathrm{SU}(2)_{L}$, respectively. The anomaly matrix is defined as

$$
t_{i a} \equiv \operatorname{tr}\left[q_{i}\left(T^{A} T^{A}\right)_{a}\right]=\frac{1}{2} \operatorname{tr}_{a}\left(q_{i}\right)
$$

where $\operatorname{tr}_{a}$ means the trace over left-handed fermions which belong to fundamental (or anti-fundamental) representation under a non-Abelian gauge symmetry of $a$. Simple cal- 
culations give

$$
t_{i a}=\frac{1}{2}\left(\begin{array}{ccc}
0 & 0 & -3 \\
0 & 0 & 9 \\
2 & -6 & 0 \\
-1 & 3 & -3 \\
-3 & 1 & -1 \\
-1 & 1 & -1 \\
-1 & 1 & -1
\end{array}\right)
$$

It is easily seen that there are no mixed non-Abelian anomalies for the hypercharge $Y / 2=-\left(q_{2} / 3+q_{3} / 2+q_{4}\right)$, and a combination $q_{7}-q_{6}$. These two $\mathrm{U}(1)$ gauge symmetries are independent in the sense of $\operatorname{tr}\left((Y / 2)\left(q_{7}-q_{6}\right)\right)=0$. There are more two independent $\mathrm{U}(1)$ gauge symmetries which are free from mixed non-Abelian anomalies,

$$
\begin{gathered}
9 q_{1}+3 q_{2}-q_{4}-q_{5}+2 q_{6}+2 q_{7}, \\
49 q_{1}+3 q_{2}-20 q_{3}+9 q_{4}+49 q_{5}-98 q_{6}-98 q_{7},
\end{gathered}
$$

but these are anomalous with the other U(1)'s.

Next, consider the anomalies among U(1)'s. The anomaly matrix is defined as

$$
t_{i j k} \equiv \operatorname{tr}\left[q_{i} q_{j} q_{k}\right]
$$

Since there are no fermion which simultaneously has three kinds of $U(1)$ charges, it is enough to consider

$$
t_{i j} \equiv t_{i j j}
$$

Straightforward calculations give

$$
t_{i j}=\left(\begin{array}{ccccccc}
0 & 0 & -6 & 3 & 9 & 3 & 3 \\
0 & 0 & 18 & -9 & -3 & -3 & -3 \\
6 & -18 & 0 & 6 & 2 & 2 & 2 \\
-3 & 9 & -6 & 0 & 0 & 0 & 0 \\
-9 & 3 & -2 & 0 & 0 & 0 & 0 \\
-3 & 3 & -2 & 0 & 0 & 0 & 0 \\
-3 & 3 & -2 & 0 & 0 & 0 & 0
\end{array}\right) .
$$

Hypercharge and $q_{7}-q_{6}$ have no anomalies with any other U(1)'s. The U(1) gauge symmetries of eqs.(31) and (32) have anomalies with $\left(q_{5}, q_{6}, q_{7}\right)$ and $\left(q_{4}, q_{5}, q_{6}, q_{7}\right)$, respectively.

The U(1) gauge symmetry of $q_{7}-q_{6}$ is anomalous with some the other $24 \mathrm{U}(1)$ 's on additional D-branes. Hypercharge is the only U(1) gauge symmetry which does not have anomaly with any other U(1)'s 5. Therefore, there are no tree-level kinetic term mixings between hypercharge and the other anomalous $\mathrm{U}(1)$ 's 6 .

The masses of the anomalous $\mathrm{U}(1)$ gauge bosons in type I theory have been explicitly calculated in Refs. [85, 86]. The mass matrix is given in D-brane basis of U(1) gauge bosons. We translate their results in case of no orientifold projection. Consider toroidal

\footnotetext{
${ }^{5}$ Since $\operatorname{tr}(Y / 2)=0$, mixed gravitational anomaly is also canceled.

${ }^{6}$ The tree-level kinetic term mixing is assumed in a phenomenological model in Ref. 84.
} 
$\left(T^{2} \times T^{2} \times T^{2}\right) / \mathbf{Z}_{N}$ orbifold in type IIB theory and two D-branes at an orbifold singularity (two D-branes can be the same one). The $\mathrm{U}(1)$ gauge symmetries on these two D-branes are labeled by $a$ and $b$. There are four cases in D-brane configurations:

1. no Dirichlet-Neumann directions with supersymmetry (D3-D3 and $\mathrm{D} 7_{i}-\mathrm{D} 7_{i}$, for example).

2. two pairs of Dirichlet-Neumann directions with supersymmetry (D3-D $7_{i}$ and $\mathrm{D} 7_{i}-\mathrm{D} 7_{j}$ with $i \neq j$, for example).

3. no Dirichlet-Neumann directions without supersymmetry (D3- $\overline{\mathrm{D} 3}$ and $\mathrm{D} 7_{i}-\overline{\mathrm{D} 7}{ }_{i}$, for example).

4. two pairs of Dirichlet-Neumann directions without supersymmetry (D3- $\overline{\mathrm{D} 7_{i}}$ and $\mathrm{D} 7_{i}-\overline{\mathrm{D} 7_{j}}$ with $i \neq j$, for example).

The mass formula for each case is given as follows in order.

$$
\begin{aligned}
& \alpha^{\prime} M_{a b}^{2}=\frac{g_{a} g_{b}}{4 \pi^{3} N} \sum_{k=0}^{N-1} \prod_{i=1,2,3}\left|2 \sin \left(\pi k v_{i}\right)\right| \operatorname{Tr}\left(\left(\gamma_{a}\right)^{k} \lambda_{a}\right)\left\{\operatorname{Tr}\left(\left(\gamma_{b}\right)^{k} \lambda_{b}\right)\right\}^{*}, \\
& \alpha^{\prime} M_{a b}^{2}=\frac{g_{a} g_{b}}{4 \pi^{3} N} \sum_{k=0}^{N-1}\left|2 \sin \left(\pi k v_{a b}\right)\right| \operatorname{Tr}\left(\left(\gamma_{a}\right)^{k} \lambda_{a}\right)\left\{\operatorname{Tr}\left(\left(\gamma_{b}\right)^{k} \lambda_{b}\right)\right\}^{*}, \\
& \alpha^{\prime} M_{a b}^{2}=\frac{1}{3} \cdot \frac{g_{a} g_{b}}{4 \pi^{3} N} \sum_{k=0}^{N-1} \prod_{i=1,2,3}\left|2 \sin \left(\pi k v_{i}\right)\right| \operatorname{Tr}\left(\left(\gamma_{a}\right)^{k} \lambda_{a}\right)\left\{\operatorname{Tr}\left(\left(\gamma_{b}\right)^{k} \lambda_{b}\right)\right\}^{*}, \\
& \alpha^{\prime} M_{a b}^{2}=-\frac{1}{3} \cdot \frac{g_{a} g_{b}}{4 \pi^{3} N} \sum_{k=0}^{N-1}\left|2 \sin \left(\pi k v_{a b}\right)\right| \operatorname{Tr}\left(\left(\gamma_{a}\right)^{k} \lambda_{a}\right)\left\{\operatorname{Tr}\left(\left(\gamma_{b}\right)^{k} \lambda_{b}\right)\right\}^{*},
\end{aligned}
$$

where $v_{i}$ is a component of $\mathbf{Z}_{N}$ twist vector $v, v_{a b}$ denotes a component of $v$ corresponding to a pair of Dirichlet-Dirichlet or Neumann-Neumann directions, $\lambda_{a}$ and $\gamma_{a}$ are ChanPaton factor and $\mathbf{Z}_{N}$ transformation matrix, respectively, and $g_{a}$ is the gauge coupling of corresponding $\mathrm{U}(1)$. These formulae guarantee that the mass matrix is Hermite: $M_{b a}^{2}=$ $\left(M_{a b}^{2}\right)^{*}$, though all the components should be real.

Since writing whole $31 \times 31$ mass matrix here is not meaningful, we examine, instead, $7 \times 7$ submatrix for $\mathrm{U}(1)$ 's which we discussed in previous subsection.

$$
\alpha^{\prime} M^{2} \simeq \frac{\sqrt{3}}{4 \pi^{3}}\left(\begin{array}{ccccccc}
3 & -\frac{1}{3} & \frac{1}{3 \sqrt{6}} & \frac{1}{6 \sqrt{3}} & -\frac{1}{2 \sqrt{3}} & \frac{1}{6 \sqrt{3}} & \frac{1}{6 \sqrt{3}} \\
-\frac{1}{3} & 3 & -\sqrt{\frac{3}{2}} & -\frac{\sqrt{3}}{2} & -\frac{1}{2 \sqrt{3}} & -\frac{1}{2 \sqrt{3}} & -\frac{1}{2 \sqrt{3}} \\
\frac{1}{3 \sqrt{6}} & -\sqrt{\frac{3}{2}} & 2 & -\frac{1}{\sqrt{2}} & -\frac{1}{3 \sqrt{2}} & -\frac{1}{3 \sqrt{2}} & -\frac{1}{3 \sqrt{2}} \\
\frac{1}{6 \sqrt{3}} & -\frac{\sqrt{3}}{2} & -\frac{1}{\sqrt{2}} & 1 & \frac{1}{3} & \frac{1}{3} & \frac{1}{3} \\
-\frac{1}{2 \sqrt{3}} & -\frac{1}{2 \sqrt{3}} & -\frac{1}{3 \sqrt{2}} & \frac{1}{3} & 1 & \frac{1}{3} & \frac{1}{3} \\
\frac{1}{6 \sqrt{3}} & -\frac{1}{2 \sqrt{3}} & -\frac{1}{3 \sqrt{2}} & \frac{1}{3} & \frac{1}{3} & 1 & \frac{1}{3} \\
\frac{1}{6 \sqrt{3}} & -\frac{1}{2 \sqrt{3}} & -\frac{1}{3 \sqrt{2}} & \frac{1}{3} & \frac{1}{3} & \frac{1}{3} & 1
\end{array}\right),
$$


where we assume for simplicity that all the gauge coupling constants are equal and of the order of unity. There is only one massless eigenstate which corresponds to hypercharge $\mathrm{U}(1)_{Y}$. The $\mathrm{U}(1)$ of $q_{7}-q_{6}$ has mass $\sqrt{3} / 6 \pi^{3} \alpha^{\prime}$, though it is anomaly free. This may be understood as the effect of higher dimensional anomalies [34, 87, 85], but anyway the gauge boson corresponding to $q_{7}-q_{6}$ is not the mass eigenstate in total $31 \mathrm{U}(1)$ 's. The total $31 \times 31$ mass matrix is calculated in the same way, but the analytic forms of its eigenvalues and eigenstates are not simple. In the limit of small gauge couplings on $\mathrm{D} 7_{2}$, $\mathrm{D} 7_{3}$ and $\overline{\mathrm{D}}_{2}$ branes (large $R$ ), mass eigenstates of 31 gauge bosons are categorized into three groups: massless $\mathrm{U}(1)_{Y}$, eight massive $\mathrm{U}(1)$ 's which dominantly couple with "our world", and 22 massive U(1)'s (six of them are very light $(\sim 1 \mathrm{MeV})$ with very small gauge couplings $\left.\left(\sim 10^{-7}\right)\right)$ which dominantly couple with "hidden world". The gauge bosons of the first and second group dominantly consist of U(1)'s on D3, D7 1 and $\overline{\mathrm{D}}_{1}$, and the gauge bosons of the third group dominantly consist of U(1)'s on the other D-branes. We consider only the second group in the following, since the contribution of gauge bosons in the third group to $S$ parameter should be very small.

The masses of eight gauge bosons in the second group, $X_{i}$ with $i=1,2, \cdots, 8$ are given by

$$
M_{X_{i}}^{2}=\frac{\sqrt{3}}{\pi}\left(M_{s}^{*}\right)^{2} f_{i}
$$

with coefficients $f_{i}$ numerically calculated as

$$
\begin{aligned}
f_{1} & \simeq 0.14, \quad f_{2} \\
f_{5} \simeq 2.3, \quad f_{6} & \simeq 2.97, \quad f_{3} \simeq 1.4, \quad f_{7} \simeq 3.4, \quad f_{8} \simeq 4.1 .
\end{aligned}
$$

Since techni-fermion condensates of eq.(27) break some U(1) gauge symmetries, these masses may receive some corrections of the order of $100 \mathrm{GeV}$. In the following we neglect these corrections to the above mass eigenvalues, because the masses produced by string effect is larger than $1 \mathrm{TeV}$ with the string scale of the order of $10 \mathrm{TeV}$. Defining the charge matrices $\tilde{Q}_{i}$ numerical calculations give

$$
\begin{gathered}
\operatorname{tr}\left(Q_{Y / 2} \tilde{Q}_{1}\right) \simeq 2.3, \quad \operatorname{tr}\left(Q_{Y / 2} \tilde{Q}_{2}\right) \simeq-0.018, \quad \operatorname{tr}\left(Q_{Y / 2} \tilde{Q}_{3}\right) \simeq-2.1 \\
\operatorname{tr}\left(Q_{Y / 2} \tilde{Q}_{4}\right) \simeq-2.3, \quad \operatorname{tr}\left(Q_{Y / 2} \tilde{Q}_{5}\right) \simeq 2.6, \quad \operatorname{tr}\left(Q_{Y / 2} \tilde{Q}_{6}\right) \simeq 2.0 \\
\operatorname{tr}\left(Q_{Y / 2} \tilde{Q}_{7}\right) \simeq-2.7, \quad \operatorname{tr}\left(Q_{Y / 2} \tilde{Q}_{8}\right) \simeq 3.2 .
\end{gathered}
$$

Therefore, all eight anomalous $\mathrm{U}(1)$ gauge bosons can have kinetic term mixing with $\mathrm{U}(1)_{Y}$ gauge boson (and with each other) at one loop level. We can understand that massless non-anomalous U(1) gauge symmetries are not necessary independent of massive anomalous $\mathrm{U}(1)$ gauge symmetries, namely $\operatorname{tr}\left(Q_{\text {non-anomalous }} Q_{\text {anomalous }}\right) \neq 0$, by explicitly and analytically checking simpler models.

\subsection{Towards a solution of $S$ parameter problem}

In case there is kinetic-term mixing between $\mathrm{U}(1)_{Y}$ gauge boson and a massive vector boson $X$ without mass mixing between $W_{3}$ and $X$, the contribution to $S$ parameter is 
given by [52]

$$
S=\frac{16}{\alpha} \frac{\left(c^{2}-\left(M_{X} / m_{Z}\right)^{2}\right) s^{2} c^{2} \omega^{2}}{\left(\left(M_{X} / m_{Z}\right)^{2}-1\right)^{2}},
$$

where $\omega$ is the coefficient of the kinetic term mixing

$$
\mathcal{L}_{\text {kin.mix }}=\omega F_{Y}^{\mu \nu} F_{X \mu \nu} .
$$

This is the second order contribution in $\omega$. The sign is always negative with $M_{X}>m_{Z}$, which is appropriate to cancel the positive contribution of technicolor, eq.(10). Note that heavy $X, M_{X} \gg m_{Z}$, quickly decouples. In case $X$ has mass mixing with $W_{3}$ there is a contribution of the first order in $\omega$ through

$$
\Pi_{3 Y}^{\prime}(0)=\Pi_{3 X}(0) \frac{g_{X}^{2}}{-M_{X}^{2}} \Pi_{Y X}^{\prime}(0)
$$

with $\Pi_{Y X}^{\prime}(0)=2 \omega[53]$. Again, heavy $X, M_{X} \gg \Pi_{3 X}(0)$, quickly decouples.

First, estimate the values of $\omega$ for eight anomalous U(1)'s. Here, we consider lowenergy effective field theory, and calculate fermion one-loop diagrams with ultraviolet cutoff. A simple estimate gives

$$
\omega_{i} \simeq-\operatorname{tr}\left(Q_{Y / 2} Q_{X_{i}}\right) \frac{g_{Y} g_{X_{i}}}{16 \pi^{2}} \ln \left(\frac{\left(M_{s}^{*}\right)^{2}}{m^{2}}\right) \simeq-\operatorname{tr}\left(Q_{Y / 2} Q_{X_{i}}\right) \frac{1}{16 \pi^{2}} \ln \left(\frac{\left(M_{s}^{*}\right)^{2}}{m^{2}}\right),
$$

where we set the string scale as ultraviolet cutoff and $m^{2}$ is an infrared cutoff which should be taken as fermion masses of the order of $1 \mathrm{GeV}$. In addition to this one-loop effect (planer open string one loop), there is string effect mediated by closed string (nonplaner open string one loop). Some explicit calculations in String Theory have already been done in Refs. [88, 89], but the subtraction of divergences due to NS-NS tadpoles (here, NS is abbreviation of Neveu-Schwarz) makes results ambiguous. For example, we can read from their results the coefficient of kinetic term mixing between D3 and $\overline{\mathrm{D}}_{1}$ branes as

$$
\omega \simeq \operatorname{tr}\left(\lambda_{D 3}\right) \operatorname{tr}\left(\lambda_{\overline{D 7}}\right) \frac{4}{(2 \pi)^{3}} \sum_{n_{1}, n_{2} \neq 0} \frac{1}{n_{1}^{2}+n_{2}^{2}},
$$

where summations are taken over winding modes in two Dirichlet-Dirichlet directions. The summation diverges due to NS-NS tadpoles, and the authors in Ref. [88] take the contribution from the first winding modes only and obtain a finite result. The procedure of tadpole resummations [90, 91, 47] may be appropriate to obtain unambiguous results. The true value could be larger than the naive estimation of eq.(47). Note that there are ambiguities in $\omega$, and we leave this problem for future works.

The second order contribution in the coefficient of kinetic term mixing between hypercharge gauge boson and lightest anomalous $\mathrm{U}(1)$ gauge boson to $S$ parameter is

$$
S \simeq-0.03
$$

where we do not consider kinetic term mixings among eight anomalous U(1)'s, for simplicity. Though the sign is appropriate to cancel the large contribution by technicolor 
dynamics, $S_{T C} \simeq 0.32$, the absolute value is one order smaller. The contribution of next lightest anomalous $\mathrm{U}(1)$ is at least two order smaller than the above value. We need a little lighter anomalous $\mathrm{U}(1)$ and/or a little larger kinetic term mixing. For example, factor three large $\omega$ is enough to satisfy the experimental constraint.

The first order contributions depend on mass mixings between $W_{3}$ and anomalous $\mathrm{U}(1)$ 's through electroweak symmetry breaking. The mass mixing is determined by the charges of technicolor condensates.

$$
m_{3 X_{i}}^{2}=m_{X_{i} 3}^{2} \simeq(100 \mathrm{GeV})^{2} \Delta Q_{X_{i}}
$$

with $\Delta Q_{X_{i}} \equiv\left[Q_{X_{i}}\left(\Psi_{L}^{I_{3}=+1 / 2} \Psi_{7_{2}}\right)-Q_{X_{i}}\left(\Psi_{L}^{I_{3}=-1 / 2} \Psi_{E}\right)\right]$, where

$$
\begin{gathered}
\Delta Q_{X_{1}}=-0.087, \quad \Delta Q_{X_{2}}=-0.011, \quad \Delta Q_{X_{3}}=0.15, \quad \Delta Q_{X_{4}}=0.18 \\
\Delta Q_{X_{5}}=-0.38, \quad \Delta Q_{X_{6}}=-0.11, \quad \Delta Q_{X_{7}}=0.019, \quad \Delta Q_{X_{i}}=-0.095 .
\end{gathered}
$$

These values of the order of $1 / 10$ reflect that each mass eigenstate has about $10 \%$ component of "hidden" anomalous U(1)'s. The contribution to $S$ parameter is

$$
S \simeq-16 \pi \sum_{i=1}^{8}\left(2 m_{3 X_{i}}^{2} \cdot \frac{1}{-M_{X_{i}}^{2}} \cdot 2 \omega_{X_{i}}\right) \simeq 0.01 .
$$

This is the same order of magnitude of the second order contribution with opposite sign. The first order contribution is not larger than the second order contribution, since the masses by electroweak symmetry breaking are much smaller than the original masses of anomalous U(1)'s. The sign of this contribution is very model dependent.

\section{Conclusions}

We have examined the possibility of dynamical electroweak symmetry breaking (technicolor) in string models by concretely analyzing a toy model mainly concentrating on the solution of $S$ parameter problem with massive anomalous U(1) gauge bosons. It has been found that the contributions of massive anomalous U(1)'s to $S$ parameter are nonnegligible, and they even have potential to cancel large technicolor contribution. Since there is no general relation between the magnitudes of technicolor and anomalous $\mathrm{U}(1)$ contributions to $S$ parameter, the cancellation, which may happen, is accidental.

It is very likely that anomalous $\mathrm{U}(1)$ gauge bosons give large contribution to $T$ parameter as well as $S$ parameter [92]. It is known that $T$ parameter is sensitive to the mechanism of quark and lepton mass generations, especially the generation of top quark mass (or top-bottom mass splitting). The string scale of the order of $10 \mathrm{TeV}$ is appropriate to generate mass of the order of $2 \pi(100)^{3} /(10000)^{2} \simeq 0.06 \mathrm{GeV}$, and it is apparently difficult to generate large top quark mass. This is more serious problem in technicolor scenario in general than $S$ parameter problem. It would be interesting to pursue the solution of this problem in string models beyond the framework of field theory.

There is a tension between dynamical electroweak symmetry breaking and light anomalous $\mathrm{U}(1)$ gauge bosons. To have light, of the order of $\mathrm{TeV}$, anomalous $\mathrm{U}(1)$ gauge bosons, 
the string scale should be less than $10 \mathrm{TeV}$. We have seen in our toy model that we need large threshold correction to the technicolor gauge coupling at the string scale so that it becomes strong at the weak scale. Larger threshold correction is required for smaller string scale. In the theoretical point of view, this scenario is very constrained. Near future collider experiments and astronomical observations will give strong constraints to this scenario.

The landscape analysis requiring additional non-Abelian gauge symmetry for dynamical electroweak symmetry breaking (without elementary Higgs doublet fields) would be interesting in the theoretical point of view.

\section{Acknowledgments}

I thank the Galileo Galilei Institute for Theoretical Physics for the hospitality and the INFN for partial support during the early stage of this work. I would like to thank Augusto Sagnotti for providing chance and support to join the workshop "New Perspectives in String Theory" at that institute. It is a great pleasure to thank Ignatios Antoniadis, Massimo Bianchi and Emilian Dudas for helpful discussions. I would like to thank Emilian Dudas for comments on the paper. This work was supported in part by INFN, and in part by the Italian MIUR-PRIN contract 2007-5ATT78.

\section{References}

[1] H. E. Haber, "Introductory low-energy supersymmetry," arXiv:hep-ph/9306207.

[2] H. P. Nilles, "Phenomenological aspects of supersymmetry," arXiv:hep-ph/9511313.

[3] H. Baer et al., "Low-energy supersymmetry phenomenology," arXiv:hep-ph/9503479.

[4] J. A. Bagger, "Weak-scale supersymmetry: Theory and practice," arXiv:hep-ph/9604232.

[5] D. J. Castano, E. J. Piard and P. Ramond, "Renormalization Group Study Of The Standard Model And Its Extensions. 2. The Minimal Supersymmetric Standard Model," Phys. Rev. D 49 (1994) 4882 [arXiv:hep-ph/9308335].

[6] M. Drees and S. P. Martin, "Implications of SUSY model building," arXiv:hep-ph/9504324.

[7] K. Inoue, A. Kakuto, H. Komatsu and S. Takeshita, "Aspects Of Grand Unified Models With Softly Broken Supersymmetry," Prog. Theor. Phys. 68 (1982) 927 [Erratum-ibid. 70 (1983) 330].

[8] N. Arkani-Hamed, A. G. Cohen and H. Georgi, "Electroweak symmetry breaking from dimensional deconstruction," Phys. Lett. B 513 (2001) 232 arXiv:hep-ph/0105239. 
[9] N. Arkani-Hamed, A. G. Cohen, E. Katz and A. E. Nelson, "The littlest Higgs," JHEP 0207 (2002) 034 arXiv:hep-ph/0206021.

[10] I. Antoniadis, "A Possible new dimension at a few TeV," Phys. Lett. B 246 (1990) 377.

[11] N. Arkani-Hamed, S. Dimopoulos and G. R. Dvali, "The hierarchy problem and new dimensions at a millimeter," Phys. Lett. B 429 (1998) 263 arXiv:hep-ph/9803315.

[12] L. Randall and R. Sundrum, "A large mass hierarchy from a small extra dimension," Phys. Rev. Lett. 83 (1999) 3370 arXiv:hep-ph/9905221.

[13] D. B. Fairlie, "Higgs' Fields And The Determination Of The Weinberg Angle," Phys. Lett. B 82 (1979) 97.

[14] N. S. Manton, "A New Six-Dimensional Approach To The Weinberg-Salam Model," Nucl. Phys. B 158 (1979) 141.

[15] Y. Hosotani, "Dynamical Mass Generation By Compact Extra Dimensions," Phys. Lett. B 126 (1983) 309.

[16] C. Csaki, C. Grojean, H. Murayama, L. Pilo and J. Terning, "Gauge theories on an interval: Unitarity without a Higgs," Phys. Rev. D 69 (2004) 055006 arXiv:hep-ph/0305237.

[17] S. Weinberg, "Implications Of Dynamical Symmetry Breaking," Phys. Rev. D 13 (1976) 974.

[18] S. Weinberg, "Implications Of Dynamical Symmetry Breaking: An Addendum," Phys. Rev. D 19 (1979) 1277.

[19] L. Susskind, "Dynamics Of Spontaneous Symmetry Breaking In The Weinberg-Salam Theory," Phys. Rev. D 20 (1979) 2619.

[20] V. A. Miransky, M. Tanabashi and K. Yamawaki, "Dynamical Electroweak Symmetry Breaking with Large Anomalous Dimension and t Quark Condensate," Phys. Lett. B 221 (1989) 177.

[21] Y. Nambu, "BOOTSTRAP SYMMETRY BREAKING IN ELECTROWEAK UNIFICATION," EFI-89-08.

[22] W. J. Marciano, "HEAVY TOP QUARK MASS PREDICTIOnS," Phys. Rev. Lett. $62(1989) 2793$.

[23] W. A. Bardeen, C. T. Hill and M. Lindner, "Minimal Dynamical Symmetry Breaking Of The Standard Model," Phys. Rev. D 41 (1990) 1647.

[24] A. Belyaev, R. Foadi, M. T. Frandsen, M. Jarvinen, F. Sannino and A. Pukhov, "Technicolor Walks at the LHC," Phys. Rev. D 79 (2009) 035006 arXiv:0809.0793 [hep-ph]]. 
[25] S. B. Giddings, S. Kachru and J. Polchinski, "Hierarchies from fluxes in string compactifications," Phys. Rev. D 66 (2002) 106006 arXiv:hep-th/0105097.

[26] G. Aldazabal, S. Franco, L. E. Ibanez, R. Rabadan and A. M. Uranga, "D = 4 chiral string compactifications from intersecting branes," J. Math. Phys. 42 (2001) 3103 arXiv:hep-th/0011073.

[27] D. Cremades, L. E. Ibanez and F. Marchesano, "Intersecting brane models of particle physics and the Higgs mechanism," JHEP 0207 (2002) 022 arXiv:hep-th/0203160.

[28] I. Antoniadis, K. Benakli and M. Quiros, "Radiative symmetry breaking in brane models," Nucl. Phys. B 583 (2000) 35 arXiv:hep-ph/0004091.

[29] N. Kitazawa, "Radiative symmetry breaking on D-branes at non-supersymmetric singularities," Nucl. Phys. B 755 (2006) 254 arXiv:hep-th/0606182.

[30] C. Bachas, "A Way to break supersymmetry," arXiv:hep-th/9503030.

[31] R. Blumenhagen, L. Goerlich, B. Kors and D. Lust, "Noncommutative compactifications of type I strings on tori with magnetic background flux," JHEP 0010 (2000) 006 arXiv:hep-th/0007024.

[32] C. Angelantonj, I. Antoniadis, E. Dudas and A. Sagnotti, "Type-I strings on magnetised orbifolds and brane transmutation," Phys. Lett. B 489 (2000) 223 arXiv:hep-th/0007090.

[33] G. Aldazabal, S. Franco, L. E. Ibanez, R. Rabadan and A. M. Uranga, "Intersecting brane worlds," JHEP 0102 (2001) 047 arXiv:hep-ph/0011132.

[34] L. E. Ibanez, F. Marchesano and R. Rabadan, "Getting just the standard model at intersecting branes," JHEP 0111 (2001) 002 arXiv:hep-th/0105155.

[35] R. Blumenhagen, B. Kors, D. Lust and T. Ott, "The standard model from stable intersecting brane world orbifolds," Nucl. Phys. B 616 (2001) 3 arXiv:hep-th/0107138.

[36] M. Cvetic, G. Shiu and A. M. Uranga, "Three-family supersymmetric standard like models from intersecting brane worlds," Phys. Rev. Lett. 87 (2001) 201801 arXiv:hep-th/0107143.

[37] M. Cvetic, G. Shiu and A. M. Uranga, "Chiral four-dimensional N = 1 supersymmetric type IIA orientifolds from intersecting D6-branes," Nucl. Phys. B 615 (2001) 3 arXiv:hep-th/0107166.

[38] C. Kokorelis, "New standard model vacua from intersecting branes," JHEP 0209 (2002) 029 [arXiv:hep-th/0205147].

[39] G. Aldazabal, L. E. Ibanez, F. Quevedo and A. M. Uranga, "D-branes at singularities: A bottom-up approach to the string embedding of the standard model," JHEP 0008 (2000) 002 [arXiv:hep-th/0005067]. 
[40] T. Higaki, N. Kitazawa, T. Kobayashi and K. j. Takahashi, "Flavor structure and coupling selection rule from intersecting D-branes," Phys. Rev. D 72 (2005) 086003 arXiv:hep-th/0504019.

[41] P. Anastasopoulos, T. P. T. Dijkstra, E. Kiritsis and A. N. Schellekens, "Orientifolds, hypercharge embeddings and the standard model," Nucl. Phys. B 759 (2006) 83 arXiv:hep-th/0605226.

[42] N. Kitazawa, "Supersymmetric composite models on intersecting D-branes," Nucl. Phys. B 699 (2004) 124 arXiv:hep-th/0401096].

[43] N. Kitazawa, "Dynamical generation of mu-terms and Yukawa couplings in intersecting D-brane models," JHEP 0411 (2004) 044 arXiv:hep-th/0403278.

[44] N. Kitazawa, T. Kobayashi, N. Maru and N. Okada, "Yukawa coupling structure in intersecting D-brane models," Eur. Phys. J. C 40 (2005) 579 arXiv:hep-th/0406115.

[45] I. Antoniadis, K. Benakli and A. Laugier, "Contact interactions in D-brane models," JHEP 0105 (2001) 044 arXiv:hep-th/0011281.

[46] S. Abel and J. Santiago, "Constraining the string scale: from Planck to Weak and back again," J. Phys. G 30 (2004) R83 [arXiv:hep-ph/0404237].

[47] N. Kitazawa, "One-loop masses of open-string scalar fields in String Theory," JHEP 0809 (2008) 049 [arXiv:0805.0824 [hep-th]].

[48] J. P. Conlon, A. Maharana and F. Quevedo, "Towards Realistic String Vacua," JHEP 0905 (2009) 109 arXiv:0810.5660 [hep-th]].

[49] R. Sundrum and S. D. H. Hsu, "Walking technicolor and electroweak radiative corrections," Nucl. Phys. B 391 (1993) 127 |arXiv:hep-ph/9206225].

[50] T. Appelquist and F. Sannino, "The Physical Spectrum of Conformal SU(N) Gauge Theories," Phys. Rev. D 59 (1999) 067702 [arXiv:hep-ph/9806409].

[51] D. K. Hong and H. U. Yee, "Holographic estimate of oblique corrections for technicolor," Phys. Rev. D 74 (2006) 015011 arXiv:hep-ph/0602177.

[52] B. Holdom, "Oblique electroweak corrections and an extra gauge boson," Phys. Lett. B 259 (1991) 329.

[53] N. Kitazawa and T. Yanagida, "A viable one-family technicolor model," Phys. Lett. B 383 (1996) 78 arXiv:hep-ph/9510228.

[54] S. Sugimoto, "Anomaly cancellations in type I D9-D9-bar system and the USp(32) string theory," Prog. Theor. Phys. 102 (1999) 685 [arXiv:hep-th/9905159].

[55] I. Antoniadis, E. Dudas and A. Sagnotti, "Brane supersymmetry breaking," Phys. Lett. B 464 (1999) 38 arXiv:hep-th/9908023. 
[56] C. Angelantonj, "Comments on open-string orbifolds with a non-vanishing B(ab)," Nucl. Phys. B 566 (2000) 126 arXiv:hep-th/9908064.

[57] G. Aldazabal and A. M. Uranga, "Tachyon-free non-supersymmetric type IIB orientifolds via brane-antibrane systems," JHEP 9910 (1999) 024 [arXiv:hep-th/9908072].

[58] C. Angelantonj, I. Antoniadis, G. D'Appollonio, E. Dudas and A. Sagnotti, "Type I vacua with brane supersymmetry breaking," Nucl. Phys. B 572 (2000) 36 arXiv:hep-th/9911081.

[59] I. Antoniadis and T. R. Taylor, "Topological masses from broken supersymmetry," Nucl. Phys. B 695 (2004) 103 arXiv:hep-th/0403293.

[60] I. Antoniadis, K. S. Narain and T. R. Taylor, "Open string topological amplitudes and gaugino masses," Nucl. Phys. B 729 (2005) 235 arXiv:hep-th/0507244].

[61] C. D. Carone, J. Erlich and M. Sher, "Holographic Electroweak Symmetry Breaking from D-branes," Phys. Rev. D 76 (2007) 015015 [arXiv:0704.3084 [hep-th]].

[62] E. Farhi and L. Susskind, "Technicolor," Phys. Rept. 74 (1981) 277.

[63] M. E. Peskin and T. Takeuchi, "A New constraint on a strongly interacting Higgs sector," Phys. Rev. Lett. 65 (1990) 964.

[64] M. E. Peskin and T. Takeuchi, "Estimation of oblique electroweak corrections," Phys. Rev. D 46 (1992) 381.

[65] G. Altarelli and R. Barbieri, "Vacuum polarization effects of new physics on electroweak processes," Phys. Lett. B 253 (1991) 161.

[66] G. Altarelli, R. Barbieri and S. Jadach, "Toward a model independent analysis of electroweak data," Nucl. Phys. B 369 (1992) 3 [Erratum-ibid. B 376 (1992) 444].

[67] C. Amsler et al. [Particle Data Group], "Review of particle physics," Phys. Lett. B 667 (2008) 1.

[68] F. Sannino and K. Tuominen, "Orientifold theory dynamics and symmetry breaking," Phys. Rev. D 71 (2005) 051901 arXiv:hep-ph/0405209].

[69] D. D. Dietrich, F. Sannino and K. Tuominen, "Light composite Higgs from higher representations versus electroweak precision measurements: Predictions for LHC," Phys. Rev. D 72 (2005) 055001 arXiv:hep-ph/0505059].

[70] R. Foadi, M. T. Frandsen, T. A. Ryttov and F. Sannino, "Minimal Walking Technicolor: Set Up for Collider Physics," Phys. Rev. D 76 (2007) 055005 arXiv:0706.1696 [hep-ph]].

[71] C. Hanhart, J. A. Pons, D. R. Phillips and S. Reddy, "The likelihood of GODs' existence: Improving the SN1987a constraint on the size of large compact dimensions," Phys. Lett. B 509 (2001) 1 arXiv:astro-ph/0102063. 
[72] S. Hannestad and G. G. Raffelt, "Stringent neutron-star limits on large extra dimensions," Phys. Rev. Lett. 88 (2002) 071301 [arXiv:hep-ph/0110067].

[73] S. Hannestad and G. G. Raffelt, "Supernova and neutron-star limits on large extra dimensions reexamined," Phys. Rev. D 67 (2003) 125008 [Erratum-ibid. D 69 (2004) 029901] arXiv:hep-ph/0304029.

[74] I. Antoniadis, C. Bachas and E. Dudas, "Gauge couplings in four-dimensional type I string orbifolds," Nucl. Phys. B 560 (1999) 93 [arXiv:hep-th/9906039].

[75] C. Bachas and C. Fabre, "Threshold Effects in Open-String Theory," Nucl. Phys. B 476 (1996) 418 arXiv:hep-th/9605028.

[76] C. P. Bachas, "Unification with low string scale," JHEP 9811 (1998) 023 arXiv:hep-ph/9807415].

[77] D. M. Ghilencea and S. Groot Nibbelink, "String threshold corrections from field theory," Nucl. Phys. B 641 (2002) 35 |arXiv:hep-th/0204094.

[78] P. Anastasopoulos, M. Bianchi, G. Sarkissian and Y. S. Stanev, "On gauge couplings and thresholds in type I gepner models and otherwise," JHEP 0703 (2007) 059 arXiv:hep-th/0612234.

[79] D. Lust and S. Stieberger, "Gauge threshold corrections in intersecting brane world models," Fortsch. Phys. 55 (2007) 427 arXiv:hep-th/0302221].

[80] N. Akerblom, R. Blumenhagen, D. Lust and M. Schmidt-Sommerfeld, "Thresholds for intersecting D-branes revisited," Phys. Lett. B 652 (2007) 53 arXiv:0705.2150 [hep-th]].

[81] K. Benakli and M. D. Goodsell, "Two-Point Functions of Chiral Fields at One Loop in Type II," Nucl. Phys. B 805 (2008) 72 [arXiv:0805.1874 [hep-th]].

[82] J. P. Conlon, "Gauge Threshold Corrections for Local String Models," JHEP 0904 (2009) 059 [arXiv:0901.4350 [hep-th]].

[83] J. P. Conlon and E. Palti, "Gauge Threshold Corrections for Local Orientifolds," arXiv:0906.1920 [hep-th].

[84] P. Anastasopoulos, F. Fucito, A. Lionetto, G. Pradisi, A. Racioppi and Y. S. Stanev, "Minimal Anomalous U(1) -prime Extension of the MSSM," Phys. Rev. D 78 (2008) 085014 arXiv:0804.1156 [hep-th]].

[85] I. Antoniadis, E. Kiritsis and J. Rizos, "Anomalous U(1)s in type I superstring vacua," Nucl. Phys. B 637 (2002) 92 arXiv:hep-th/0204153.

[86] P. Anastasopoulos, "Anomalous U(1)s masses in non-supersymmetric open string vacua," Phys. Lett. B 588 (2004) 119 arXiv:hep-th/0402105]. 
[87] C. A. Scrucca, M. Serone and M. Trapletti, "Open string models with ScherkSchwarz SUSY breaking and localized anomalies," Nucl. Phys. B 635 (2002) 33 arXiv:hep-th/0203190|.

[88] S. A. Abel and B. W. Schofield, "Brane-antibrane kinetic mixing, millicharged particles and SUSY breaking," Nucl. Phys. B 685 (2004) 150 arXiv:hep-th/0311051.

[89] S. A. Abel, M. D. Goodsell, J. Jaeckel, V. V. Khoze and A. Ringwald, "Kinetic Mixing of the Photon with Hidden U(1)s in String Phenomenology," JHEP 0807 (2008) 124 [arXiv:0803.1449 [hep-ph]].

[90] E. Dudas, G. Pradisi, M. Nicolosi and A. Sagnotti, "On tadpoles and vacuum redefinitions in string theory," Nucl. Phys. B 708 (2005) 3 [arXiv:hep-th/0410101].

[91] N. Kitazawa, "Tadpole Resummations in String Theory," Phys. Lett. B 660 (2008) 415 [arXiv:0801.1702 [hep-th]].

[92] D. M. Ghilencea, L. E. Ibanez, N. Irges and F. Quevedo, "TeV-Scale Z' Bosons from D-branes," JHEP 0208 (2002) 016 [arXiv:hep-ph/0205083]. 
Table 1: D-brane configuration for twisted R-R tadpole cancellations.

\begin{tabular}{|c|c|c|c|c|c|}
\hline$\left(\mathrm{I}_{1}, \mathrm{I}_{2}, \mathrm{I}_{3}\right)$ & D3 & $\mathrm{D} 7_{1}$ & $\mathrm{D} 7_{2}$ & $\mathrm{D} 7_{3}$ & $\overline{\mathrm{D}}_{1}$ \\
\hline$\left(\mathrm{II}_{1}, \mathrm{I}_{2}, \mathrm{I}_{3}\right)$ & $\overline{\mathrm{D} 3}^{(1)}$ & $\mathrm{D} 7_{1}^{\prime}$ & $\mathrm{D} 7_{2}$ & $\mathrm{D} 7_{3}$ & \\
\hline$\left(\mathrm{III}_{1}, \mathrm{I}_{2}, \mathrm{I}_{3}\right)$ & $\overline{\mathrm{D} 3}^{(2)}$ & $\mathrm{D} 7_{1}^{\prime \prime}$ & $\mathrm{D} 7_{2}$ & $\mathrm{D} 7_{3}$ & \\
\hline$\left(\mathrm{I}_{1}, \mathrm{II}_{2}, \mathrm{I}_{3}\right)$ & $\mathrm{D} 3^{(1)}$ & $\mathrm{D} 7_{1}$ & ${\overline{\mathrm{D}} 7_{2}^{\prime}}^{\prime}$ & $\mathrm{D} 7_{3}$ & $\overline{\mathrm{D}}_{1}$ \\
\hline$\left(\mathrm{I}_{1}, \mathrm{III}_{2}, \mathrm{I}_{3}\right)$ & $\mathrm{D} 3^{(2)}$ & $\mathrm{D} 7_{1}$ & $\overline{\mathrm{D} 7_{2}^{\prime \prime}}$ & $\mathrm{D} 7_{3}$ & $\overline{\mathrm{D}}_{1}$ \\
\hline$\left(\mathrm{II}_{1}, \mathrm{II}_{2}, \mathrm{I}_{3}\right)$ & & $\mathrm{D} 7_{1}^{\prime}$ & $\overline{\mathrm{D}}_{2}^{\prime}$ & $\mathrm{D} 7_{3}$ & \\
\hline$\left(\mathrm{II}_{1}, \mathrm{III}_{2}, \mathrm{I}_{3}\right)$ & & $\mathrm{D} 7_{1}^{\prime}$ & $\overline{\mathrm{D}}_{2}^{\prime \prime}$ & $\mathrm{D} 7_{3}$ & \\
\hline$\left(\mathrm{III}_{1}, \mathrm{II}_{2}, \mathrm{I}_{3}\right)$ & & $\mathrm{D} 7_{1}^{\prime \prime}$ & ${\overline{\mathrm{D}} 7_{2}^{\prime}}^{\prime}$ & $\mathrm{D} 7_{3}$ & \\
\hline$\left(\mathrm{III}_{1}, \mathrm{III}_{2}, \mathrm{I}_{3}\right)$ & & $\mathrm{D} 7_{1}^{\prime \prime}$ & $\overline{\mathrm{D} 7_{2}^{\prime \prime}}$ & $\mathrm{D} 7_{3}$ & \\
\hline$\left(\mathrm{I}_{1}, \mathrm{I}_{2}, \mathrm{II}_{3}\right)$ & D3' & $\mathrm{D} 7_{1}$ & $\mathrm{D} 7_{2}$ & $\mathrm{D} 7_{3}^{\prime}$ & $\overline{\mathrm{D}}_{1}$ \\
\hline$\left(\mathrm{II}_{1}, \mathrm{I}_{2}, \mathrm{II}_{3}\right)$ & $\overline{\mathrm{D}}^{(3)}$ & $\mathrm{D} 7_{1}^{\prime}$ & $\mathrm{D} 7_{2}$ & $\mathrm{D} 7_{3}^{\prime}$ & \\
\hline$\left(\mathrm{III}_{1}, \mathrm{I}_{2}, \mathrm{II}_{3}\right)$ & $\overline{\mathrm{D} 3}^{(4)}$ & $\mathrm{D} 7_{1}^{\prime \prime}$ & $\mathrm{D} 7_{2}$ & $\mathrm{D} 7_{3}^{\prime}$ & \\
\hline$\left(\mathrm{I}_{1}, \mathrm{II}_{2}, \mathrm{II}_{3}\right)$ & $\mathrm{D} 3^{(3)}$ & $\mathrm{D} 7_{1}$ & $\overline{\mathrm{D}}_{2}^{\prime}$ & $\mathrm{D} 7_{3}^{\prime}$ & $\overline{\mathrm{D}}_{1}$ \\
\hline$\left(\mathrm{I}_{1}, \mathrm{III}_{2}, \mathrm{II}_{3}\right)$ & $\mathrm{D} 3^{(4)}$ & $\mathrm{D} 7_{1}$ & $\overline{\mathrm{D} 7_{2}^{\prime \prime}}$ & $\mathrm{D} 7_{3}^{\prime}$ & $\overline{\mathrm{D}}_{1}$ \\
\hline$\left(\mathrm{II}_{1}, \mathrm{II}_{2}, \mathrm{II}_{3}\right)$ & & $\mathrm{D} 7_{1}^{\prime}$ & $\overline{\mathrm{D}}_{2}^{\prime}$ & $\mathrm{D} 7_{3}^{\prime}$ & \\
\hline$\left(\mathrm{II}_{1}, \mathrm{III}_{2}, \mathrm{II}_{3}\right)$ & & $\mathrm{D} 7_{1}^{\prime}$ & $\overline{\mathrm{D} 7_{2}^{\prime \prime}}$ & $\mathrm{D} 7_{3}^{\prime}$ & \\
\hline$\left(\mathrm{III}_{1}, \mathrm{II}_{2}, \mathrm{II}_{3}\right)$ & & $\mathrm{D} 7_{1}^{\prime \prime}$ & $\overline{\mathrm{D}}_{2}^{\prime}$ & $\mathrm{D} 7_{3}^{\prime}$ & \\
\hline$\left(\mathrm{III}_{1}, \mathrm{III}_{2}, \mathrm{II}_{3}\right)$ & & $\mathrm{D} 7_{1}^{\prime \prime}$ & $\overline{\mathrm{D} 7}{ }_{2}^{\prime \prime}$ & $\mathrm{D} 7_{3}^{\prime}$ & \\
\hline$\left(\mathrm{I}_{1}, \mathrm{I}_{2}, \mathrm{III}_{3}\right)$ & D3" & $\mathrm{D} 7_{1}$ & $\mathrm{D} 7_{2}$ & $\mathrm{D} 7_{3}^{\prime \prime}$ & $\overline{\mathrm{D}}_{1}$ \\
\hline$\left(\mathrm{II}_{1}, \mathrm{I}_{2}, \mathrm{III}_{3}\right)$ & $\overline{\mathrm{D} 3}^{(5)}$ & $\mathrm{D} 7_{1}^{\prime}$ & $\mathrm{D} 7_{2}$ & $\mathrm{D} 7_{3}^{\prime \prime}$ & \\
\hline$\left(\mathrm{III}_{1}, \mathrm{I}_{2}, \mathrm{III}_{3}\right)$ & $\overline{\mathrm{D} 3}^{(6)}$ & $\mathrm{D} 7_{1}^{\prime \prime}$ & $\mathrm{D} 7_{2}$ & $\mathrm{D} 7_{3}^{\prime \prime}$ & \\
\hline$\left(\mathrm{I}_{1}, \mathrm{II}_{2}, \mathrm{III}_{3}\right)$ & $\mathrm{D} 3^{(5)}$ & $\mathrm{D} 7_{1}$ & ${\overline{\mathrm{D}} 7_{2}^{\prime}}^{\prime}$ & $\mathrm{D} 7_{3}^{\prime \prime}$ & $\overline{\mathrm{D}}_{1}$ \\
\hline$\left(\mathrm{I}_{1}, \mathrm{III}_{2}, \mathrm{III}_{3}\right)$ & $\mathrm{D} 3^{(6)}$ & $\mathrm{D} 7_{1}$ & $\overline{\mathrm{D} 7}{ }_{2}^{\prime \prime}$ & $\mathrm{D} 7_{3}^{\prime \prime}$ & $\overline{\mathrm{D}}_{1}$ \\
\hline$\left(\mathrm{II}_{1}, \mathrm{II}_{2}, \mathrm{III}_{3}\right)$ & & $\mathrm{D} 7_{1}^{\prime}$ & ${\overline{\mathrm{D}} 7_{2}^{\prime}}^{\prime}$ & $\mathrm{D} 7_{3}^{\prime \prime}$ & \\
\hline$\left(\mathrm{II}_{1}, \mathrm{III}_{2}, \mathrm{III}_{3}\right)$ & & $\mathrm{D} 7_{1}^{\prime}$ & $\overline{\mathrm{D} 7}{ }_{2}^{\prime \prime}$ & $\mathrm{D} 7_{3}^{\prime \prime}$ & \\
\hline$\left(\mathrm{III}_{1}, \mathrm{II}_{2}, \mathrm{III}_{3}\right)$ & & $\mathrm{D} 7_{1}^{\prime \prime}$ & $\overline{\mathrm{D}}_{2}^{\prime}$ & $\mathrm{D} 7_{3}^{\prime \prime}$ & \\
\hline$\left(\mathrm{III}_{1}, \mathrm{III}_{2}, \mathrm{III}_{3}\right)$ & & $\mathrm{D} 7_{1}^{\prime \prime}$ & $\overline{\mathrm{D} 7_{2}^{\prime \prime}}$ & $\mathrm{D} 7_{3}^{\prime \prime}$ & \\
\hline
\end{tabular}


Table 2: Massless fermion modes at $\left(\mathrm{I}_{1}, \mathrm{I}_{2}, \mathrm{I}_{3}\right)$ singularity. The gauge symmetries on additional D-branes are considered as global symmetries in this table. The gauge symmetries on $\mathrm{D} 7_{i}$-brane are described as $\mathrm{U}(1)_{i}^{\prime}$ with $i=1,2,3$. The contents above the horizontal line are associated with open strings between two D-branes of our world, and those below the horizontal line are associated with open strings between our D-branes and additional D-branes.

\begin{tabular}{cccccccccc}
$\mathrm{U}(3)_{\mathrm{TC}}$ & $\mathrm{U}(3)_{c}$ & $\mathrm{U}(2)_{L}$ & $\mathrm{U}(1)$ & $\mathrm{U}(1)_{1}^{\prime}$ & $\mathrm{U}(1)_{2}^{\prime}$ & $\mathrm{U}(1)_{3}^{\prime}$ & & $\mathrm{U}(1)_{Y}$ & \\
\hline \hline 1 & $3^{*}$ & 1 & +1 & 0 & 0 & 0 & $\times 3$ & $-2 / 3$ & $u_{R}^{c}$ \\
1 & 3 & $2^{*}$ & 0 & 0 & 0 & 0 & $\times 3$ & $+1 / 6$ & $q_{L}$ \\
1 & 1 & 2 & -1 & 0 & 0 & 0 & $\times 3$ & $+1 / 2$ & $\tilde{h}^{\prime \prime}$ \\
1 & $3^{*}$ & 1 & 0 & +1 & 0 & 0 & & $+1 / 3$ & $d_{R}^{c}$ \\
1 & 1 & 2 & 0 & -1 & 0 & 0 & & $-1 / 2$ & $l_{L}$ \\
1 & $3^{*}$ & 1 & 0 & 0 & +1 & 0 & & $+1 / 3$ & $d_{R}^{c}$ \\
1 & 1 & 2 & 0 & 0 & -1 & 0 & & $-1 / 2$ & $l_{L}$ \\
1 & $3^{*}$ & 1 & 0 & 0 & 0 & +1 & & $+1 / 3$ & $d_{R}^{c}$ \\
1 & 1 & 2 & 0 & 0 & 0 & -1 & & $-1 / 2$ & $l_{L}$ \\
$3^{*}$ & 1 & 2 & 0 & 0 & 0 & 0 & & $-1 / 2$ & $\Psi_{L}^{I_{3}}$ \\
3 & 1 & 1 & -1 & 0 & 0 & 0 & & +1 & $\Psi_{E}$ \\
3 & 1 & 1 & 0 & -1 & 0 & 0 & $\times 3$ & 0 & $\Psi_{7_{1}}^{i}$ \\
3 & 1 & 1 & 0 & 0 & -1 & 0 & & 0 & $\Psi_{7_{2}}$ \\
3 & 1 & 1 & 0 & 0 & 0 & -1 & & 0 & $\Psi_{7_{3}}$ \\
\hline $3^{*}$ & 1 & 1 & 0 & 0 & 0 & 0 & $\times 2$ & 0 & $\Psi_{7_{2}^{\prime}}^{I_{3}}$ \\
$3^{*}$ & 1 & 1 & 0 & 0 & 0 & 0 & $\times 2$ & 0 & $\Psi_{7_{2}^{\prime \prime}}^{I_{2}^{\prime \prime}}$ \\
3 & 1 & 1 & 0 & 0 & 0 & 0 & & 0 & $\Psi_{7_{3}^{\prime}}$ \\
3 & 1 & 1 & 0 & 0 & 0 & 0 & & 0 & $\Psi_{7^{\prime \prime}}^{\prime}$ \\
$3^{*}$ & 1 & 1 & 0 & 0 & 0 & 0 & $\times 2$ & 0 & $\Psi_{L}^{\prime I_{3}}$ \\
3 & 1 & 1 & 0 & 0 & 0 & 0 & & 0 & $\Psi_{E}^{\prime}$ \\
$3^{*}$ & 1 & 1 & 0 & 0 & 0 & 0 & $\times 2$ & 0 & $\Psi_{L}^{\prime \prime} I_{3}$ \\
3 & 1 & 1 & 0 & 0 & 0 & 0 & & 0 & $\Psi_{E}^{\prime \prime}$ \\
1 & 1 & 1 & 0 & +1 & 0 & 0 & $\times 12$ & 0 & \\
1 & 1 & 1 & 0 & -1 & 0 & 0 & $\times 4$ & 0 & \\
1 & 1 & 1 & 0 & 0 & +1 & 0 & $\times 6$ & 0 & \\
1 & 1 & 1 & 0 & 0 & -1 & 0 & $\times 4$ & 0 & \\
1 & 1 & 1 & 0 & 0 & 0 & +1 & $\times 2$ & 0 & \\
\hline \hline
\end{tabular}

